\title{
Jean-Claude Guédon
}

Professeur de littératures comparées, Université de Montréal

(1984)

\section{"L’idéologie comme mode d'appropriation de la science"}

Un document produit en version numérique par Jean-Marie Tremblay, bénévole, professeur de sociologie au Cégep de Chicoutimi

Courriel: jmt_sociologue@,videotron.ca

Site web pédagogique : http://www.uqac.ca/jmt-sociologue/

Dans le cadre de la collection: "Les classiques des sciences sociales"

Site web: http://www.uqac.ca/Classiques_des_sciences_sociales

Une collection développée en collaboration avec la Bibliothèque

Paul-Émile-Boulet de l'Université du Québec à Chicoutimi

Site web: http://bibliotheque.uqac.uquebec.ca/index.htm 
Cette édition électronique a été réalisée par Jean-Marie Tremblay, bénévole, professeur de sociologie au Cégep de Chicoutimi à partir de :

"L'idéologie comme mode d'appropriation de la science". Un article publié dans l'ouvrage sous la direction de Claude Savary et Claude Panaccio, L'idéologie et les stratégies de la raison. Approches théoriques, épistémologiques et anthropologiques, pp. 149-167. Montréal: Éditions Hurtubise HMH ltée, 1984, 236 pp. Collection Brèches.

Avec l'autorisation formelle de M. Jean-Claude Guédon, professeur de littératures comparées, Université de Montréal, le 6 mai 2004.

89 Courriel : jean.claude.guedon@umontreal.ca

Polices de caractères utilisée :

Pour le texte: Times, 12 points.

Pour les citations : Times 10 points.

Pour les notes de bas de page : Times, 10 points.

Édition électronique réalisée avec le traitement de textes Microsoft Word 2001 pour Macintosh.

Mise en page sur papier format

LETTRE (US letter), 8.5' x 11'”)

Édition numérique réalisée le 4 novembre 2004 à Chicoutimi, Ville de Saguenay, province de Québec, Canada.

\section{Fait avec}

Macintosh 
Le titre de cet essai peut s'interpréter de deux manières qu'il s'agit de bien distinguer. En effet le petit mot « de » peut recéler soit un génitif, soit un ablatif, c'est-à-dire deux perspectives inverses. Dans le premier cas, c'est la science qui se fait approprier tandis que, dans le deuxième cas, c'est la science qui effectue l'appropriation grâce à l'idéologie.

Le deuxième terme de l'alternative au sein du titre a été déjà traité par Georges Canguilhem dans un essai intitulé. "Qu'est-ce qu'une idéologie scientifique ${ }^{1}$ ? » Rappelons brièvement les points principaux de son argumentation qui cherche à cerner aussi précisément que possible le rôle épistémologique d'un concept aux allures pour le moins paradoxales. En fait Canguilhem parle même de monstruosité parce qu'il est devenu difficile de parler d'idéologie, non pas comme d'une théorie de la genèse des idées, théorie élaborée au début du XIXe siècle par Maine de Biran, Destutt de Tracy et d'autres, mais, à la suite de Marx, comme titre général de «tout système d'idées produit comme effet d'une situation initialement condamnée à méconnaître son rapport réel au réel ${ }^{2} \gg$.

Pour contourner l'aspect monstrueux, c'est-à-dire apparemment contradictoire, de l'expression «idéologie scientifique », Canguilhem propose de distinguer contenu et fonction. Le contenu serait idéologique au sens habituel du terme, puisqu'une idéologie scientifique consisterait surtout en formations discursives à prétention de théorie, en représentations de relations entre phénomènes, en axes stables de commentaires visant l'expérience vécue ${ }^{3}$. Ce contenu en forme de catalogue qu'offre Georges Canguilhem est donc parfaitement compatible avec la notion d'idéologie chez Marx telle que l'histoire des sciences nous la donne ${ }^{4}$, d'abord parce qu'il s'agit d'un catalogue un peu éclec-

1 G. CANGUILHEM, «Qu'est-ce qu'une idéologie scientifique? » in Idéologie et rationalité dans l'histoire des sciences de la vie, Paris, Vrin, 1977, pp. 33-45. Cet article est repris de Organon (Varsovie), ${ }^{\circ} 7$ (1970).

Ibid., p. 36.

Ibid., p. 35.

4 Personnellement, je n'ai aucune difficulté avec la représentation de Marx que donne Canguilhem et donc j'aurais pu être tenté d'écrire que les contenus de l'idéologie donnés par Canguilhem sont compatibles avec la définition de l'idéologie selon Marx. Mais ce 
tique, et non d'une définition visant à garantir l'unité essentielle du concept d'idéologie scientifique, ensuite parce que ce contenu s'oppose au « rapport réel au réel » de trois manières: sa prétention de théorie, sa plus ou moins grande cohérence et sa relative durabilité. Prétention, cohérence floue et vie transitoire: voilà les trois points grâce auxquels on peut stigmatiser le contenu de toute idéologie scientifique.

Reste la fonction d'une idéologie scientifique. Pour aborder cette question, Canguilhem reprend une formule de l'historien des sciences Suchodolski, formule qui revient à dire qu'une histoire des sciences conçue comme une histoire des vérités scientifiques est irréalisable parce que ce dernier terme renferme une contradiction interne ${ }^{5}$. En effet, l'énoncé de vérités scientifiques, vérités prises ici dans un sens à la fois général et absolu, ne peut mener à une histoire, mais seulement à un historique, de la même manière que des énoncés logiques déduits d'énoncés antérieurs peuvent n'avoir d'historique que le fait de se succéder à un rythme irrégulier et même imprévisible. Ainsi, pour contourner ce dilemme, Canguilhem change de terrain et, plutôt que de parler de vérités scientifiques, il met en avant l'opposition entre science authentique et science inauthentique en soulignant d'ailleurs que parler de science authentique, c'est se doter d'un titre qui correspond à une revendication de dignité 6 . Ensuite il place le rapport entre science authentique et science inauthentique sous le signe générale de l'éviction. L'éviction, nous rappelle-t-il, c'est la dépossession juridique d'un bien acquis de bonne foi ${ }^{7}$. La question qui se pose alors est simple: si l'histoire des sciences doit s'occuper des sciences authentiques, ce que personne ne conteste, doit-elle exclure, tolérer ou bien revendiquer l'histoire des rapports d'éviction de l'inauthentique par l'authentique 8 ? La fonction d'une science inauthentique, autrement dit une idéologie scientifique, c'est d'usurper temporairement le terrain d'une science authentique.

Arrivé à ce stade de son argument, Canguilhem nous livre quelques précisions supplémentaires sur l'idéologie scientifique, et en particulier qu'il ne s'agit ni d'une fausse science, ni d'une fausse conscience ${ }^{9}$. Dans le dernier cas,

qui importe en fait, c'est non pas le rapport de la théorie de l'idéologie chez Canguilhem avec la théorie correspondante chez Marx, mais plutôt la manière de se situer de Canguilhem par rapport à sa propre représentation de Marx. En d'autres termes, Canguilhem est prêt à dire: je me représente le contenu de l'idéologie chez Marx comme « $\mathrm{x} », \ll \mathrm{y} », \ll \mathrm{z}$ » et $\mathrm{j}$ 'assume ce contenu.

5 G. CANGUILHEM, op. cit., note 1, p. 34. Le texte de Canguilhem, lu à Varsovie en octobre 1969, répond au texte que Suchodolski avait lu à la fin d'août 1968 à Paris. Voir B. SUCHODOLSKI, «Les Facteurs du développement de l'histoire des sciences », XIIe Congrès international d'histoire des sciences - Colloques-textes des rapports, Paris, Albin Michel, 1968, pp. 27-38.

G. CANGUILHEM, op. cit., note 1, p. 33.

Ibid.

Ibid.

Ibid., p. 39. 
l'idéologie se réduirait tout simplement à une idéologie politique tout à fait banale, tandis qu'une fausse science, parce qu'elle ne rencontre jamais le faux ${ }^{10}$, n'a jamais à faire face à des situations où il lui faut changer ou renoncer à quelque partie d'elle-même que ce soit. Par conséquent, une fausse science n'a pas d'histoire, déclare Canguilhem, à l'inverse d'une idéologie scientifique qui en a une, et surtout une fin.

C'est d'ailleurs par référence au problème de sa fin que l'idéologie scientifique peut voir se préciser sa fonction d'usurpation face à la science authentique qui lui correspond. "Une idéologie scientifique trouve une fin, quand le lieu qu'elle occupait dans l'encyclopédie du savoir se trouve investi par une discipline qui fait la preuve, opérativement, de la validité de ses normes de scientificité ${ }^{11}$. » D'où le rapport d'usurpation introduit en début d'analyse par Canguilhem: mais cette usurpation est curieuse si l'on considère que l'occupant présumé est accusé d'avoir pris la place de quelque chose qui n'est pas encore constitué. En fait, l'idéologie scientifique ne devient usurpatrice qu'au moment même où elle perd ses apparences de scientificité pour être reléguée au rang d'idéologie. À l'assignation du terme d'idéologie correspond donc l'accusation d'usurpation, correspondance aussi temporelle que fonctionnelle. En d'autres mots, l'idéologie scientifique perd toute son efficacité discursive au moment même où on lui assigne et fait reconnaître son statut d'idéologie. Les idéologies scientifiques sont donc les scories nécessairement trouvées dans le sillage de l'histoire des sciences, histoire comprise ici dans le sens que Canguilhem donne à ce mot.

La fonction d'usurpation caractérise donc l'idéologie scientifique à l'instant précis de sa défaite, mais elle ne suffit pas à rendre compte de toutes les fonctions de ce concept. En effet, le catalogue encyclopédique des savoirs ne peut s'identifier que par des sciences authentiques et par des sciences inauthentiques ou idéologies. Ceci veut dire que, sans la présence d'idéologies scientifiques, certaines rubriques de ce catalogue encyclopédique demeureraient invisibles. Par conséquent, tout en faisant obstacle aux sciences authentiques, les idéologies scientifiques préparent leur propre perte en contribuant à identifier le lieu où va se déployer la nouvelle science authentique. Inversement, les idéologies scientifiques ne pourraient se constituer sans «loucher ${ }^{12} »$ vers des sciences authentiques situées dans des secteurs voisins

10 Canguilhem est, sur ce point, très proche de Popper.

11 Ibid., p. 39. Canguilhem précise du même coup que l'idéologie scientifique n'est pas une anti-science, mais une non-science, puisqu'elle a l'ambition explicite d'être une science. Ainsi, là où Suchodolski visait à interpréter l'évolution historique des sciences par une dialectique entre vrai et faux, Canguilhem, en passant à l'opposition authentique / inauthentique, se donne les moyens de raffiner l'analyse en introduisant un troisième élément entre science et anti-science, celui de non-science.

12 G. CANGUILHEM, op. cit., note 1, p. 44. Canguilhem dit également que l'idéologie est déportée par rapport au site que viendra tenir la science. (Ibid., p. 39.) 
et déjà constituées. L'entrelacs science-idéologie scientifique est donc crucial pour rendre compte de la dynamique conceptuelle des sciences elles-mêmes.

Au total, donc, l'arraisonnement d'un domaine de savoir par une nouvelle science authentique, processus engendré par et, en même temps, contre l'idéologie scientifique en place, se traduit par une redéfinition profonde de ce domaine de savoir car « ce que la science trouve n'est pas ce que l'idéologie donnait à chercher ${ }^{13} \gg$. En fait, les idéologies scientifiques correspondent à des percées prématurées de la quête scientifique, à cette sorte « d'antériorité de l'aventure intellectuelle ${ }^{14}$ » qui est le pendant d'une thèse fondamentale selon laquelle la science n'évolue jamais paisiblement. En d'autres termes, la science n'évolue pas de manière continue et les idéologies ne sont que les gouvernements provisoires de territoires mal conquis.

À la fin de son essai, Canguilhem distingue les idéologies scientifiques des idéologies des scientifiques ${ }^{15}$. Ces dernières ne seraient que des énoncés produits par les savants quand, par exemple, ils thématisent leur méthode de recherche ou quand ils parlent de science et de son rôle dans la culture. De plus, elles renversent la perspective des idéologies scientifiques, puisqu'elles mobilisent certaines facettes de l'activité scientifique à des fins d'ordre idéologique au sens banal du terme.

Au total, l'analyse de Canguilhem maintient fermement la ligne de démarcation entre science et idéologie, même s'il conçoit l'existence d'un rapport entrelacé et conflictuel entre ces deux ordres présumés du savoir. Or, c'est justement cette démarcation entre science et idéologie qu'il s'agit de remettre en question ici en se demandant s'il est possible d'imaginer une activité scientifique réelle qui puisse se dérouler sans accorder un rôle essentiel à l'idéologie.

En posant cette dernière question, il convient de ne pas se laisser entraîner par une autre thèse, celle qui consiste à télescoper science et idéologie. Cette thèse a été soutenue par divers philosophes, en particulier Marcuse et Habermas, lorsqu'ils montrent que la science agit comme idéologie chaque fois qu'elle légitime des processus de domination ${ }^{16}$. La question soulevée ici est tout autre: entre le rapport extérieur et l'identification totale existe une situation intermédiaire où la science, tout en évinçant l'idéologie scientifique comme le montre Canguilhem, ne le ferait pas au nom d'une différence essentielle de nature, mais se construirait sa propre nature au détour de chaque conflit dont la fin se marquerait par l'identification d'une idéologie nouvelle.

Ibid., p. 40.

Ibid., p. 38.

Ibid., pp. 43-44.

J. HABERMAS, La Technique et la science comme idéologie, Paris, Denoël-Gonthier, 1978. (Trad. franç. de J.-R. Ladmiral suivant l'édition allemande de 1968, p. 37.) 
Avant de poursuivre, un dernier détour par Althusser peut s'avérer utile. En effet, ce dernier retraduit l'analyse de Canguilhem en d'autres termes. En divisant ce qu'il appelle la philosophie spontanée des savants (P.S.S.) en deux éléments distincts, 1 et 2, Althusser reconduit la division entre la science et son extérieur ${ }^{17}$. Selon Althusser, l'élément 1, nécessairement matérialiste, est souvent recouvert ou masqué par l'élément 2 qui substitue ses énoncés à ceux d'une philosophie proprement scientifique. Ainsi, là où l'élément 1 livre des concepts comme « objet », «théorie » et « méthode », l'élément 2 y substitue subrepticement d'autres termes comme «données de l'expérience », «modèles » et « techniques $18 \gg$.

D'Althusser on peut retenir que, même si en pratique la grande majorité des scientifiques voient l'élément 1 de leur philosophie spontanée fortement contaminé par l'élément 2, en théorie la possibilité d'envisager une P.S.S. purement matérialiste demeure intacte. Althusser peut ainsi sauver la démarcation entre science et idéologie et maintenir que tout savant qui disposerait d'une P.S.S. réellement matérialiste verrait sa vie de savant simplifiée de beaucoup. Or, la thèse explorée ici prétend, au contraire, que si un savant était complètement libre de toute idéologie, il ne pourrait plus fonctionner comme savant.

$$
* * *
$$

Pour mener à bien une telle démonstration, il s'agit d'abord de prouver que la science est essentiellement complexe, complexité comprise en ce sens que, en plus d'éléments repris de ses états antérieurs, la science ne peut évoluer que si elle incorpore des éléments qui lui sont extérieurs. Pour mieux comprendre ce que peut impliquer une telle formule, il sera utile de faire un nouveau détour par le projet, déjà ancien, d'une histoire des sciences conçue comme une histoire des idées scientifiques. Je pense tout particulièrement aux études dont le modèle est donné par les travaux de A. O. Lovejoy, d'une part, et d'A. Koyré, d'autre part, études qui ont longtemps dominé le champ de l'histoire des sciences en Amérique du Nord comme en Europe.

Arthur Lovejoy, l'auteur du livre célèbre, The Great Chain of Being ${ }^{19}$, divise son plan d'analyse en deux parties. Il s'agit d'abord de repérer une doctrine ou système de pensée, souvent philosophique, comme le néo-plato-

17 L. ALTHUSSER, Philosophie et philosophie spontanée des savants, Paris, Maspero, 1974, pp. 100-101. (La première édition remonte à 1967.)

18 Ibid., pp. 103-104.

19 Arthur O. LOVEJOY, The Great Chain of Being. A Study of the History of an Idea. (D'abord publié en 1936 chez Harvard University Press.) 
nisme, afin d'en isoler les éléments idéels ou idées unitaires (unit ideas). Ces idées unitaires sont dotées d'un statut tout à fait analogue à celui des éléments chimiques, ce que Lovejoy explicite d'ailleurs dans l'introduction de son ouvrage ${ }^{20}$ et qu'il exploite ensuite en comparant la doctrine de n'importe quel philosophe à un composé chimique, composé instable de surcroît ${ }^{21}$, et dont la nouveauté repose, en général, beaucoup plus sur son organisation interne que sur l'apparition d'éléments nouveaux.

Si Lovejoy s'appuie sur la chimie pour parler d'idées unitaires, il passe en revanche à la médecine pour les utiliser: il s'agit alors de mener une sorte d'étude épidémiologique de la diffusion des idées unitaires en repérant les manifestations de ces entités dans la pensée collective de grands groupes humains ${ }^{22}$. Et ce qui est intéressant chez Lovejoy, c'est qu'il ne structure pas son domaine de recherche à partir de catégories elles-mêmes dépendantes de l'histoire, telle la science ou, pour être encore plus précis, la chimie; au contraire, Lovejoy fait reposer sa démarche sur des collections de textes aussi représentatives que possible de toute une époque.

Pour justifier cette diffusion générale de certaines idées unitaires dans pratiquement tout le tissu culturel d'une époque, Lovejoy, en bon platonicien, invoque l'influence que ces idées pouvait exercer sur les esprits ou consciences des êtres humains. Bref, l'histoire des idées est fondée sur un objet particulier, l'idée unitaire, et un processus de diffusion, l'influence. Son objectif final est très ambitieux puisqu'il ne vise rien de moins que l'élucidation des dimensions psychologiques des processus grâce auxquels des modifications dans la vogue et l'influence des idées ont pu survenir ${ }^{23}$.

Ce qui est intéressant dans la démarche de Lovejoy, c'est le rapport très particulier qui semble s'établir entre l'être humain et les idées unitaires. En bref, chaque texte qui incorpore une idée unitaire particulière est perçu à la fois comme attestation d'une présence, ce qui permet à l'historien de tracer son fil le long de l'axe du temps, mais aussi comme élément dynamique. En effet le contact d'un texte incorporant une idée unitaire avec l'esprit ou la conscience d'un lecteur entraîne un phénomène de transmission: sous l'effet de l'influence qu'exercerait cette idée sur son esprit, le lecteur, transformé en écrivain, est conduit à la réutiliser, parfois fort loin de son domaine d'utilisation antérieur. L'idée unitaire, par conséquent, loin d'être le produit d'un cerveau, mène au contraire une carrière autonome, autonomie qui n'est interrompue que temporairement quand elle parasite en quelque sorte l'esprit humain et ainsi obtient sa retranscription dans un texte nouveau. Ces dernières

Ibid., p. 3.

Ibid.

Ibid., p. 19.

Ibid., p. 20. 
métaphores ne sont pas de Lovejoy, mais elles mettent clairement en évidence, me semble-t-il, les caractéristiques curieuses attribuées à ces entités.

Avant de revenir à cette notion d'influence, passons très rapidement à la méthode d'Alexandre Koyré. Celui-ci écrit une histoire qui, en fait, est une sorte de biographie de l'esprit humain en marche vers la vérité ${ }^{24}$. Mais ce mouvement chez Koyré se fonde sur une cause beaucoup plus concrète, en apparence du moins, que la mystérieuse influence de Lovejoy: l'effet d'histoire résulte en effet, chez Koyré, du fait qu'une question, quelle qu'elle soit, est reprise tôt ou tard par un autre auteur. Mais cette reprise est conçue comme une sorte de discussion qui se perpétuerait à travers le temps et à l'échelle de l'humanité. En fait, pour Koyré, l'histoire des idées scientifiques évolue un peu comme un interminable dialogue platonicien, ce dont témoigne la citation suivante:

Les étapes principales de la route qui mène du Monde clos à l'Univers infini, apparaissent très clairement dans les oeuvres de quelques grands penseurs... C'est d'eux et de leurs oeuvres que nous occuperons ici, et la tâche nous sera facilitée par le fait qu'elles se réfèrent l'une à l'autre et se présentent à nous comme des démarches successives d'une discussion serrée ${ }^{25}$.

Même si Koyré privilégie les grands penseurs alors que Lovejoy étend ses recherches aux auteurs de deuxième et troisième catégories, ces deux auteurs se rapprochent par leur platonisme personnel, ce qui amène Koyré à examiner comment certaines conceptions philosophiques sont nécessairement antérieures aux conceptions scientifiques puisque ce sont elles qui informent au sens philosophique et fort du terme, ces dernières. Ainsi, Koyré et Lovejoy sont amenés à considérer la conception d'idées scientifiques comme étant la résultante de plusieurs idées antérieures, dont l'origine ne se situe pas nécessairement dans le domaine scientifique ${ }^{26}$. Seulement, ce même platonisme les amène à faire usage de processus de transmission ou mythiques (les grands penseurs dialoguent virtuellement entre eux à travers les âges), ou mystérieux (l'influence).

24 A. KOYRÉ, Études galiléennes, Paris, Hermann, 1955, p. 11. (La première édition remonte à 1939.)

25 A. KOYRÉ, Du monde clos à l'univers infini, Paris, PUF, 1962, p. 4. C'est moi qui souligne.

26 Ces remarques devraient suffire pour montrer que l'internalisme de Koyré n'est pas aussi simple qu'on le présente parfois. Pour Koyré, ce n'est pas l'autonomie absolue de la science par rapport au social qu'il revendique, c'est l'autonomie de la science par rapport à certains éléments sociaux comme la structure de classe, le domaine appliqué, l'économie en général, et ainsi de suite; en revanche les présomptions philosophiques (platonisme, archimédisme, aristotélisme, etc.) sont placées au tout premier rang de ses analyses et en constituent même un des éléments essentiels. 
Mythiques ou mystérieux, ces processus (ou d'autres analogues dans d'autres traditions historiques) jouent un rôle important en ce sens qu'ils tentent de rendre compte d'un fait essentiel, à savoir comment penser l'évolution de la science comme élaboration d'éléments antérieurs à elle-même et recomposés au sein d'un nouvel ensemble avec des éléments hétérogènes à ellemême? En d'autres termes, même si le dialogue et l'influence sont des processus vides de sens, leur présence signale l'existence d'un besoin - celui d'un processus qui donnerait les mêmes résultats mais en se dotant, en plus, d'une matérialité plus observable, plus directement reliée aux diverses formes de comportement observées chez les scientifiques.

Avant d'examiner quel pourrait être ce processus, notons tout de même que la notion d'influence a la vie dure, comme en témoigne ce bref historique. Elle sévit encore dans un livre récent de Maurice Crosland sur Gay-Lussac ${ }^{27}$, livre qui n'est certainement pas exceptionnel de ce point de vue. Pourtant, et ce dès le début du siècle, la notion d'influence subissait de rudes assauts. Ce mot qui ne désigne qu'une ignorance ou une hypothèse, comme le dit Paul Valéry, fut également critiqué en U.R.S.S. par J. Tynianov dans le contexte d'une réévaluation de ce que pourrait être une histoire littéraire digne de ce nom ${ }^{28}$. Plus près de nous, Michel Foucault a également rejeté l'influence en particulier et l'histoire des idées en général en élaborant le concept de discours comme pratique et comme objet ${ }^{29}$. Comme Tynianov, Foucault considère que l'influence recouvre en fait une analogie entre divers textes, qui ne peut être spécifiée que si elle est replacée au sein d'une série précise et bien identifiée ${ }^{30}$.

Pour bien illustrer le fonctionnement habituel de la notion d'influence, faisons donc appel à un texte qui, curieusement, n'utilise jamais ce mot et pourtant ne parle pas d'autre chose. Ce texte, emprunté à Maurice Crosland, explicite sans le vouloir la notion d'influence et se révèle donc particulièrement utile pour en débusquer les assises.

En étudiant la vie et l'œuvre de Gay-Lussac, on ne peut manquer d'être frappé tôt ou tard par certaines ressemblances avec son grand compatriote Lavoisier. Il n'y eut aucun contact direct entre eux puisque Gay-Lussac n'avait que quinze ans et vivait au fond de la province quand le plus grand chimiste de France fut mené à la guillotine installée sur la Place de la Révolution à Paris. Ce n'est que plusieurs années après la Terreur que Gay-Lussac vit naître son intérêt pour la chimie et ce fut alors par le truchement de Berthollet et de l'École Polytechnique. Cependant, grâce à Berthollet, l'aîné des convertis à la nouvelle chimie, un grand nombre d'idées de Lavoisier furent transmises à l'aspirant chimiste. D'ailleurs l'amitié et les intérêts communs que

27 M. CROSLAND, Gay-Lussac - Scientist and Bourgeois, Londres, Cambridge University Press, 1978, passim mais en particulier le dernier paragraphe de cette étude, pp. 261-262.

28 J. TYNIANOV, "De l'évolution littéraire », in Théorie de la littérature. Textes des formalistes russes, Paris, Seuil, 1965, publié sous la direction de Tzvetan Todorov, pp. 120-137. La critique de Tynianov date de 1927.

29 M. FOUCAULT, L'Archéologie du savoir, Paris, Gallimard, 1969, pp. 177-183.

30 M. Foucault et Tynianov utilisent le mot « série ». 
partageaient Lavoisier et Berthollet et Laplace les plaçaient presque en relations de famille et, pourrait-on prétendre, leur protégé de la génération suivante appartenait à l'École de Lavoisier par descendance directe ${ }^{31}$.

Même si le mot influence est absent de ce texte, on reconnaît aisément le processus auquel il renvoie: si Gay-Lussac appartient à l' «École» de Lavoisier, ce n'est pas au terme d'un apprentissage sérieux d'un certain savoir imparti selon une méthode pédagogique. «École » ici est le terme symétrique d'influence en ce sens que l'on peut dire que si Lavoisier influence en quelque manière Gay-Lussac, alors celui-ci appartient, en quelque sorte, à l'école de celui-là. Mais «École» ne suffit pas à circonscrire le mode d'action de l'influence; le terme cité en fait ajoute deux autres dimensions à cette école: l'Église (qui apparaît par le truchement des «convertis») et la famille.

Cette prolifération de termes donnés comme équivalents au mot influence est intéressante, parce qu'elle démontre en fait l'extraordinaire insuffisance de ce mot: l'influence est censée faire passer des « idées » d'un esprit à un autre, mais ce mécanisme de passage demeure bien énigmatique. Aussi pour en dissimuler le côté mystérieux convient-il d'en parler avec un vocabulaire emprunté, et ce n'est certainement pas un hasard, aux institutions chargées de la reproduction de la société. Seulement ce vocabulaire est complètement métaphorique: tour se passe comme si école, famille et religion conspiraient pour reproduire une idée d'une génération à l'autre, mais en fait il ne reste que la notion d'influence et l'établissement des traditions qu'elle est censée permettre, comme l'avait déjà noté Tynianov il y a plus d'un demi-siècle ${ }^{32}$. Influences et traditions s'appellent d'ailleurs réciproquement pour construire l'effet de continuité qui constitue la clef de voûte de toute histoire des idées, et des idées scientifiques en particulier.

Le détour fait par l'histoire des idées scientifiques nous a donc révélé que la science se construisait non seulement en évinçant des idéologies scientifiques, mais également à partir d'éléments disparates dont certains appartiennent en propre au passé de la science tandis que d'autres proviennent de secteurs plus ou moins éloignés de la science et, en tout cas, extérieurs à elle. Ces leçons, bien qu'empruntées à des études dont les failles apparaissent clairement quand on les considère sous le point de vue des mécanismes qui créent l'effet dynamique propre à l'histoire, ne peuvent être rejetées à la légère du point de vue factuel. La dynamique historique telle que la conçoivent Koyré ou Lovejoy ne convainc plus guère, mais leurs analyses de textes scientifiques demeurent encore largement valables. Ces analyses révèlent constamment que ce qui se donne comme science, se nourrit sans cesse d'éléments extrascientifiques sans que l'on sache pour autant où se situe précisément la limite entre le scientifique et le non-scientifique. Par conséquent,

M « CROSLAND, op. cit., note 27, p. 44.

J. TYNIANOV, op. cit., note 29, p. 136. 
l'information contenue dans les études de Koyré et de Lovejoy et de leurs nombreux disciples, constitue un indice documentaire important pour l'enjeu de cet essai.

Arrivé à ce stade de l'analyse, on peut imaginer de mieux préciser le rapport science-idéologie scientifique en cherchant un processus qui pourrait se substituer à celui, trop éthéré, d'influence. Pour ce faire, deux détours préalables doivent encore être accomplis. Le premier, très court, revient à limiter, au moins temporairement, notre regard aux textes et seulement à eux. Cette injonction méthodique, qui renvoie à la conceptualisation du discours comme objet chez Foucault, permet d'examiner la structure des textes visés. Et ce que dit de ces textes Julia Kristeva, en pensant surtout aux textes littéraires, s'applique a fortiori à des textes scientifiques: parce qu'il «se construit comme mosaïque de citations, tout texte est absorption et transformation d'un autre texte. À la place de la notion d'intersubjectivité s'installe celle d'intertextualité ${ }^{33}$. » Bien sûr, c'est cette notion d'intertextualité qui permet de créer les séries où les analogies peuvent se spécifier.

Au-delà du problème de l'intertextualité se situe la question d'un sujet adéquat pour rendre compte de l'absorption et de la transformation d'un texte dans un autre et la question du type de processus lié à ces absorption et transformation textuelles. À cette double question, un sociologue donne au moins quelques éléments de réponse; il s'agit de Pierre Bourdieu. Il écrit, en effet: «Le champ scientifique est toujours le lieu d'une lutte plus ou moins inégale entre des agents inégalement pourvus de capital symbolique, donc inégalement en mesure de s'approprier le produit du travail scientifique ${ }^{34}$... » Seulement cette réponse exige quelques explications, ne serait-ce que parce que le terme «s'approprier » n'apparaît que comme produit final d'un réseau dense de concepts associés. D'abord la notion de champ: Bourdieu nous dit par ailleurs qu'ils «se présentent à l'appréhension synchronique comme des espaces structurés de positions (ou de postes) dont les propriétés dépendent de leur position dans ces espaces ${ }^{35} \ldots$ » Que la coquetterie propre au style de Bourdieu et qui consiste en la répétition de mots auxquels on assigne des sens différents, ne fasse pas obstacle: la métaphore du champ de force est celle qu'il faut garder en tête, champ de force dont les pôles sont situés dans les institutions et à laquelle il faut ajouter la notion de lutte. Le champ de force est un champ de rapports de force et donc un champ conflictuel. Bourdieu, d'emblée, nous arrache au cadre serein d'une situation socio-historique où seuls des idées ou des textes existeraient et circuleraient, pour nous planter fermement

[Mot grec]. Recherches pour une sémanalyse, Paris, Seuil, 1969, p. 146.

P. BOURDIEU, «La Spécificité du champ scientifique et les conditions sociales du progrès de la raison », Sociologie et sociétés, vol. 7, no 1 (1975), p. 91.

35 P. BOURDIEU, Questions de sociologie, Paris, éditions de Minuit, 1980, p. 113. Cette définition, en fait de novembre 1976, date d'un exposé de Bourdieu fait à l'École normale supérieure devant des philologues et historiens de la littérature. 
dans une arène. Que cette arène ait des limites floues, c'est évident, mais je laisserai cet aspect de la question de côté pour le moment.

Dans le champ, il y a des agents qu'il ne faut pas confondre avec des individus, mais cette partie de l'individu que Bourdieu appelle un « habitus », lequel habitus renferme plusieurs éléments comme un ensemble de techniques, de références et un ensemble de croyances ${ }^{36}$. « Le sujet de l'œuvre c'est donc un habitus en relation avec un poste, c'est-à-dire avec un champ», écrira Bourdieu, précisément au sujet d'hommes de lettres, d'auteurs ${ }^{37}$. Quant au capital symbolique, il est constitué de connaissances acquises, de capacités personnelles et de leurs rapports avec des postes (ou positions) dans le champ.

Reste l'acte d'appropriation auquel se réfère Bourdieu sans pourtant en expliciter le sens. C'est sur ce point que je vais tenter et de retrouver le travail qu'accomplissait l'influence de manière idéaliste (par opposition à la matérialiste) et de compléter l'analyse qu'offre Bourdieu. Pour ce faire, mieux vaut commencer par quelques illustrations historiques.

Plutôt que de renvoyer à un exemple déjà développé ailleurs ${ }^{38}$, j'aimerais plutôt Mobiliser plusieurs épisodes concrets où l'on peut montrer des types d'appropriation de la science qui sont légitimes du point de vue scientifique. Il ne s'agit donc pas de montrer comment la science a été détournée de ses objectifs scientifiques pour servir des buts extra-scientifiques, ce qui correspondrait à l'analyse de l'élément 2 de la philosophie spontanée des savants selon Althusser; il ne s'agit pas non plus de montrer comment, dans certaines circonstances sociales particulières, les scientifiques entraînent la recherche scientifique le long de voies qui, rétrospectivement, semblent parfaitement aberrantes, et ce même si de tels cas sont beaucoup plus troublants dans la mesure où l'on peut se demander ce que le lyssenkisme aurait voulu dire s'il avait réussi à se perpétuer non pas une vingtaine d'années, mais deux ou trois siècles ${ }^{39}$. Mais ni lyssenkisme, ni science nazie ne constituent de bons exemples de ce que je recherche précisément parce que, après coup, ces sciences ont été invalidées.

En revanche les analyses que mènent Thackray et Morrell de certaines dimensions de la science anglaise ${ }^{40}$ sont beaucoup plus difficiles à balayer $\mathrm{du}$

Ibid., p. 144.

Ibid., p. 212.

J.-C. GUÉDON, «À qui appartient la science? », Chercheurs, vol. 6, no 4 (octobre 1980), pp. 15-16.

39 Sur le lyssenkisme, il existe toute une série de textes, mais l'une des interprétations les plus intéressantes de ce phénomène est celle de Dominique Lecourt, Lyssenko - Histoire réelle d'une science prolétarienne, Paris, Maspero, 1976, passim.

40 J.B. MORRELL et A. THACKRAY, Gentlemen of Science - The Beginning of the British Association for the Advancement of Science, Cambridge, Cambridge University Press, 1981, pp. 21-29. 
revers de la main. En faisant l'histoire des quatorze premières années de la British Association for the Advancernent of Science, J.B. Morrell et A. Thackray sont amenés à en analyser les fonctions sociales: la science vise à connaître la nature et y reconnaître l'œuvre de Dieu. De ce fait, elle constitue un lieu idéal où peuvent s'unifier des fractions de noblesse et de bourgeoisie qui autrement éprouvent beaucoup de mal à communiquer entre elles. Or, cette unification a pris de l'importance car, face aux résistances croissantes des ouvriers et des paysans, les classes dominantes doivent offrir un front uni. La science, en quelque sorte offre le terrain neutre propice à une telle réconciliation.

La thèse des deux auteurs ne devient réellement intéressante que lorsqu'ils commencent à examiner les choix opérés par l'oligarchie qui contrôle de fait la B.A.A.S., choix qui privilégient certains types d'activités scientifiques à l'exclusion d'autres. Si, de ce point de vue, le refus d'admettre la phrénologie peut nous sembler justifié parce que, rétrospectivement, cette spécialité s'est trouvée de toute façon disqualifiée, il n'en va pas de même pour les statistiques. En effet, et sous prétexte que ces recherches entraînaient la B.A.A.S. sur des terrains où le consensus social difficilement établi entre la bourgeoisie et la noblesse aurait pu éclater, les statistiques se voient bloquées pendant très longtemps. Autre choix également très significatif, la B.A.A.S. privilégie et d'ailleurs finance des recherches fortement empiriques tels que des calculs permettant de standardiser des observations d'étoiles opérées à partir de lieux différents, ou des tables de marées valables pour toute la côte anglaise. En revanche, les formalisations mathématiques et l'élaboration de modèles théorisés sont peu appréciées en physique et sont délaissées au profit de procédures purement expérimentales. Ainsi, la théorie de la propagation ondulatoire de la lumière et la controverse qui suivit l'énonciation de cette théorie en Grande-Bretagne éclairent parfaitement le préjugé expérimental qu'entretient la B.A.A.S.

$$
* * *
$$

La notion de choix introduite plus haut est encore imprécise; beaucoup plus précise serait la question suivante: la science qui s'est développée en Grande-Bretagne est-elle le produit d'un champ où les agents ont été amenés à s'approprier de manière particulière (à spécifier) les produits de la science telle qu'elle leur était disponible? Ces actes d'appropriation ont été accomplis par des scientifiques et, rétrospectivement, leur rôle apparaît comme celui, fort classique, de transmetteurs et de créateurs de savoir. Mais en fait, ils se sont approprié ce qu'ils pouvaient et, ce faisant, ils ont transformé les matériaux disponibles selon les règles propres à leur champ d'action. Or ces règles peuvent nous sembler ou très normales (cas de l'exclusion de la phrénologie), ou, au contraire, très étranges (cas du refus des statistiques), ou encore 
empreintes d'un préjugé excessif en faveur de l'empirisme. Et ces jugements que nous passons au XXe siècle sur les activités de scientifiques du XIXe siècle reposent en fait sur le recoupement partiel entre les caractéristiques du champ scientifique des « scientists ${ }^{41}$ » de la B. A. A. S. et celles du ou des champs scientifiques actuels qui, de manière composite, contribuent à engendrer en nous une image unifiée de la science. Par exemple, faire des mesures empiriques de marées peut être lu, rétrospectivement, comme une activité scientifique légitime parce que sanctionnée par certaines activités scientifiques actuelles; mais, et de manière beaucoup plus intéressante, ce peut être lu comme l'appropriation d'une activité effectuée de manière à privilégier le consensus établi en refoulant tout intérêt pour les fondations théoriques d'une telle démarche. Or, la thèse de ce texte, c'est que tout acte d'appropriation opéré dans un champ scientifique se trouvera nécessairement à la résultante de facteurs aussi hétérogènes que ceux qui apparaissent dans l'exemple résumé ci-dessus.

Cela dit, je ne suis pas encore au bout de mes peines. En effet, si quelque chose comme un début de démonstration s'élabore quant au problème de l'élaboration de la science à partir d'éléments hétérogènes, scientifiques et non-scientifiques, je n'ai pas encore précisé le sens de scientifique et de nonscientifique et, surtout, je n'ai pas encore précisé le sens que je donne au mot idéologie. En fait ces deux questions se réduisent à une seule si je fais l'hypothèse, dure, qu'il y a identité totale entre le non-scientifique et l'idéologique. Reste alors à identifier la démarcation entre science et non-science à laquelle me conduit tout ce cheminement, ou plutôt le principe permettant l'existence de la démarcation.

Si l'on reprend certains aspects de la thèse de Morrell et de Thackray telle que je me l'approprie ici, ce point peut probablement être résolu assez facilement. Il s'agit en effet de comparer la phrénologie avec les statistiques. Même si la résistance de la B.A.A.S. à l'égard de ces deux domaines n'a pas détruit l'un ou l'autre, elle a certainement contribué à les faire reculer dans l'ordre hiérarchique implicite qui existe toujours entre domaines scientifiques, cette hiérarchie pouvant d'ailleurs être interprétée comme un effet de champ. À la limite l'exclusion de ces domaines remet en cause leur légitimité. Dans un cas, les statistiques, cette légitimité se reconstruit ailleurs et avec succès, si bien que ce domaine a reconquis sa légitimité, tandis que l'inverse s'est passé pour la phrénologie. Cette manière de considérer les actes d'exclusion de la B.A.A.S. montre que cette distinction science et non-science, cette démarcation n'est que le résultat de rapports de force qui, de part en part, sont traversés par des contingences d'ordre historique. Par conséquent la démarcation

41 C'est au sein de cette association que se crée le mot «scientist», justement pour démarquer les membres de la B.A.A.S d'autres praticiens liés à d'autres institutions comme la Société royale. 
science / non-science ou, au sens dur du terme, science / idéologie n'est pas liée à une quelconque nature transhistorique de la science; elle se construit à partir des luttes et enjeux spécifiques à un champ scientifique en un lieu et un temps donnés. Mais simultanément, ,cette démarcation est inévitable, car elle est un effet de la lutte lorsque celle-ci se traduit en victoire ou défaite locale. Les actes d'inclusion et d'exclusion doivent donc être examinés avec soin, puisque ce sont eux qui construisent les démarcations entre science et nonscience, démarcations immédiatement retraduites en termes qui gomment leur caractère contingent en les faisant passer pour une conséquence de la supériorité naturelle de la nature de la science sur celle de la non-science. Mais la lutte continue et les prochaines étapes de l'appropriation contribuent à faire se déplacer ces lignes de démarcation et donc à en montrer l'aspect profondément historique.

La science évolue donc par appropriation d'éléments qui lui sont localement hétérogènes, et ces actes d'appropriation relèvent d'une série d'enjeux disparates en ce sens qu'ils incorporent simultanément des éléments cognitifs et sociaux. Mais cette hétérogénéité est elle-même fonction de l'acte d'appropriation particulier qui fait évoluer l'activité scientifique: après l'acte d'appropriation, l'hétérogène n'apparaît comme tel que par effet d'une exclusion; autrement, il disparaît dans le scientifique. Par conséquent, la science ne peut s'opposer simplement à une idéologie; en fait elle se redéfinit constamment par rapport à un envers dont, et c'est là l'essentiel de sa force, elle contrôle les modalités d'existence. Canguilhem, par conséquent, a raison de dire que l'idéologie scientifique est évincée de sa position par la science, mais il a tort de penser que cette éviction se fait au moyen de la supériorité inévitable de la nature de la science, science qui gagnerait toutes ses victoires en faisant «ses preuves par la cohérence et l'intégration de ses résultats 42 ».

En fait, la nature de la science fait essentiellement partie de chaque conflit et intervient dans ce conflit, non pas comme garant de la victoire, mais comme enjeu, si bien qu'elle peut changer et que, par conséquent, son opposition à toute idéologie ne peut-être que localement polémique et non relever d'une quelconque théorie universelle de la démarcation.

Fin du texte

42 G. CANGUILHEM, op. cit., note 1, p. 42. 